\title{
Éditorial
}

\section{Excellence territoriale}

$\Gamma$ es forces de la mondialisation incitent les collectivités à une ouverture accentuée de leur territoire aux influences économiques, culturelles et sociales dites globales. Pour bien tirer leur épingle du nouveau jeu, cependant, les collectivités doivent miser sur des effets de fermeture qui puissent leur permettre de se positionner adéquatement sur l'échiquier planétaire. Les exemples de succès périphériques en cette matière sont nombreux, en prenant l'étiquette de milieux innovateurs, districts fertiles et autres systèmes territoriaux d'innovation et de production. En réalité, l'ouverture accentuée à l'économie mondiale semble profiter davantage aux territoires qui misent sur leur spécificité, leurs avantages, leurs forces par l'entremise d'un degré de fermeture approprié pour le soutien aux activités économiques, sociales et culturelles. Un territoire peut s'ouvrir davantage, notamment en misant sur de nouvelles infrastructures de transport et de communication. Il peut aussi miser sur des mesures reliées à la fermeture du territoire telles que le renforcement culturel, la dotation de services spécialisés, l'appropriation de leviers économiques, la fertilisation de l'innovation. En contexte régional du Québec, le mélange d'ouverture et de fermeture semble réussir à un bon degré en Beauce, dans les Bois-Francs, dans le Bas-du-Fleuve mais aussi à Sacré-Cœur, Sept-Îles, Baie-Saint-Paul, Maskinongé et Lac-Mégantic.

Il est intéressant de constater à cet effet que l'économie du vaste territoire historique de Saguenay s'avère traditionnellement caractérisée par sa grande ouverture à la demande mondiale d'abord de fourrures, ensuite de bois-d'œuvre, de fromages, de pâtes, de papier, d'aluminium, de lait. Les avant-postes d'occupation territoriale tels que Chicoutimi, Dolbeau, Chibougamau, Sept-Îles ont physiquement servi d'appui à cette liaison entre une vaste périphérie et le marché mondial. Chicoutimi ne fut longtemps qu'un simple relais de cette ouverture régionale qui drainait les profits vers Québec, Paris et ensuite Londres. Le véritable développement de ce lieu (et de sa région) fut amorcé par la rétention partielle des fruits de l'exploitation de sa vaste périphérie grâce à des actions de fermeture territoriale. À partir de 1838, en effet, l'établissement de colons, de bûcherons, de commerces, de services spécialisés, de coopératives, d'institutions religieuses, de services santé et de maisons d'éducation a permis de retenir en région une partie de la richesse créée qui auparavant sortait directement du territoire, sans générer de développement. Un certain cumul local et régional de capitaux fut alors possible par les classes forestière, agricole, religieuse et d'affaires. Capitaux endogènes qui, associés aux apports exogènes, ont stimulé la croissance par l'entremise d'investissements bien ciblés. Investissements qui permirent de cumuler encore davantage de richesses territoriales. L'équilibre du développement continu fut alors atteint dans cette oasis nordique qu'est le Saguenay-Lac-Saint-Jean.

Or, l'équilibre entre ouverture et fermeture acquis à divers degrés selon les périodes illustre des ratées depuis deux décennies. Une plus grande ouverture de l'économie régionale a actuellement lieu sous deux formes principales. D'abord, l'apport technologique qui, malgré la hausse de la production, élimine les emplois et les salaires qui y sont associés, notamment dans l'agriculture, l'aluminium, le bois d'œuvre, les pâtes et papier. La vague très actuelle affecte encore plusieurs usines et unités de production de services. Ensuite, l'intégration des activités économiques régionales par des grands groupes nationaux et internationaux s'est beaucoup accentuée dans le bois d'œuvre, l'agroalimentaire, l'alimentation, le commerce, la restauration, les services aux entreprises, etc. Phénomène d'intégration qui, du coup, accélère encore davantage la fuite des profits et des rentes vers les sièges sociaux extérieurs, généralement dans les grands centres urbains. À ces effets négatifs qui s'additionnent, les nouveaux efforts de fermeture territoriale tels que la mise en place de fonds de développement, l'incubation de PME, la R\&D... apparaissent insuffisants, d'autant plus que la dotation d'équipements publics 
est arrivée à saturation. Tant et si bien que le taux de chômage affiche un niveau très élevé comparativement à celui du Québec et du Canada. La population régresse, notamment de 5,5\% entre 1994 et 2004. Bref, malgré certains investissements privés bénéfiques, l'économie régionale subit une rupture de l'équilibre qui l'a pourtant si bien servie jadis. D'autres solutions à ce récent déséquilibre deviennent nécessaires afin de mieux contenir le déclin économique amorcé au début des années 1980 et qui s’accentue actuellement.

À cet effet, nul doute que l'ouverture encore plus grande du territoire pourrait offrir des effets en matière de développement. Nous pensons notamment à la prospection intégrée de technologies, d'investisse- ments, de promoteurs ainsi que d'expertise et de savoir-faire. De nouvelles mesures coordonnées de démarchage et d'accueil doivent être envisagées, à commencer par notre image régionale perçue. Signalons aussi la nécessité d'une meilleure maîtrise collective des technologies de l'information et de la communication (TIC) par l'entremise, notamment, d'un portail régional intégré d'hyperliens. L’exploration encore plus extensive de la périphérie nordique avec les technologies récentes s'avère aussi une solution intéressante, entre autres par la veille proactive et la conception de projets. À cet effet de conception, le noyau d'entreprises du secteur «tertiaire-moteur », bien présent dans les centres urbains de la région, doit à notre avis devenir le fer de lance en étant un véritable créneau d'excellence nordique.

\section{Composantes de l'excellence territoriale 02}

\begin{tabular}{|c|c|c|c|}
\hline \multicolumn{4}{|c|}{ Ouverture } \\
\hline \multirow{2}{*}{$\begin{array}{c}\text { Oasis } \\
\text { nordique }\end{array}$} & $\begin{array}{l}\text { Exploration } \\
\text { Prospection } \\
\text { Image régionale }\end{array}$ & $\begin{array}{l}\text { Maîtrise des TIC } \\
\text { Tertiaire moteur } \\
\text { Veille collective }\end{array}$ & \multirow{2}{*}{$\begin{array}{l}\text { Centres } \\
\text { services }\end{array}$} \\
\hline & $\begin{array}{l}\text { Capital humain } \\
\text { Transformation 2e et 3e } \\
\text { Protection des ressources }\end{array}$ & $\begin{array}{l}\text { Réappropriation financière } \\
\text { Décentralisation } \\
\text { Actifs relationnels }\end{array}$ & \\
\hline
\end{tabular}

Fermeture

Du côté de la fermeture territoriale, les efforts portés récemment au soutien à la $2^{\mathrm{e}}$ et $3^{\mathrm{e}}$ transformation des ressources naturelles s'inscrivent évidemment dans le bon esprit. Le Saguenay-Lac-Saint-Jean nécessite certes d'importantes mesures de protection et de renouvellement de ses ressources naturelles. Aussi, des efforts radicaux de réappropriation de ses moyens de production (scieries, centrales hydroélectriques, usines, fromageries, fermes, etc.) et de ses marchés intérieurs de biens et services doivent être envisagés impérativement. Face aux fuites financières qui s'accélèrent dramatiquement, il apparaît essentiel d'inventer un mécanisme institutionnel approprié pour retenir davantage en région la richesse créée dont les surplus s'inscrivent sous diverses formes (épargne, fonds de pension, profits, redevances...). Toujours sous l'angle de la fermeture territoriale, la décentralisation de certaines unités de grandes entreprises, de sociétés d’État et de certains ministères s'avère aussi une voie pertinente à emprunter. La succursale de production CGI, le Centre fédéral de données fiscales, le Centre québécois de recherche et de développement de l'aluminium, le Centre des technologies de l'aluminium représentent à cet effet de beaux exemples à illustrer à Hydro-Québec, à Alcan, au ministère des Ressources naturelles et à Développement Économique Canada. Car ces organisations peuvent bénéficier au Saguenay-Lac-Saint-Jean d'une structure de coûts avantageuse, d'une proximité de l'objet de leur mission et d'une bonne qualité de vie pour leurs ressources humaines.

À cet effet de ressources humaines, la fertilisation de ce capital immatériel s'avère aussi un enjeu tout à fait stratégique dans la région 02. Mis à part les classiques tels que l'éducation, la formation et la R\&D sur lesquels la région doit évidemment encore miser, quatre composantes nous apparaissent essentielles à interpel- 
ler. D’abord, la promotion fortement accentuée de la culture régionale (apprentissage des langues, arts, voyages, spectacles, stages et aventures extra régionaux, échanges internationaux, etc.), y compris la culture industrielle (transferts, mobilité, réseautage, innovation...) et la culture organisationnelle (démocratie, concertation, partenariats...). Ensuite, la médiation systématique des relations de travail encore trop instables malgré les gains évidents de flexibilité vers la création d'une véritable classe ouvrière intermédiaire tout en protégeant la classe ouvrière supérieure. En outre, la région doit miser sur le renouvellement continu de son élite décisionnelle grâce à une implication sociale élargie, stimulée par la possibilité d'ascension sociale de la relève qualifiée. Les jeunes, les autochtones, les immigrants, les retraités sont à mobiliser particulièrement. Finalement, l'apprentissage collectif nécessite d'être stimulé davantage par la mise en interaction accrue de diverses sources de savoir et de savoir-faire.

Nul doute que les défenseurs du statu quo souligneront le caractère utopique de ces nouvelles mesures d'ouverture et de fermeture de la région. À la triple lumière de la théorie du développement régional, de l'expérience acquise au Saguenay-Lac-Saint-Jean et des initiatives originales très actuelles qui émergent ici et là, nous avançons qu'elles sont faisables. Selon notre lecture régionale, les acteurs du Saguenay-LacSaint-Jean doivent en ce sens travailler collectivement à la mise en œuvre (ou à la poursuite) des mesures d'ouverture et de fermeture précitées par l'entremise de nouvelles initiatives originales pouvant s'inscrire, lorsque nécessaire, en discontinuité avec les actions actuelles. Par l'apprentissage et la créativité, ce travail collectif fera émerger d'autres mesures peut-être encore plus audacieuses grâce à la mobilisation de l'énergie, de la détermination et de l'imagination qui caractérisent fortement, trop souvent ailleurs, la population régionale en exode.

\section{Marc-Vrbain Proulx}

Université du Québec à Chicoutimi

\section{Note}

1 Selon les Études économiques régionales de Desjardins, vol. 3, $\mathrm{n}^{\circ}$ 2, le Saguenay-Lac-Saint-Jean a affiché une perte de 3300 emplois au premier semestre de 2005 en regard de la même période en 2004. 


\section{Publicité}

\section{CRDT}

